Linköping Studies in Science and Technology

Licentiate Thesis No. 1680

\title{
Synthesis and characterization of
}

\section{Ga-containing}

\section{MAX phase thin films}

\author{
Andrejs Petruhins
}

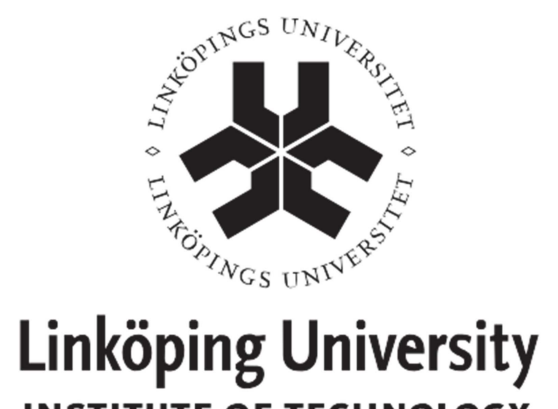

INSTITUTE OF TECHNOLOGY

\author{
Materials Design \\ Thin Film Physics Division \\ Department of Physics, Chemistry, and Biology (IFM) \\ Linköping University \\ SE-581 83 Linköping, Sweden
}


(C) Andrejs Petruhins

ISBN: 978-91-7519-224-6

ISSN: 0280-7971

Printed by LiU-Tryck

Linköping, Sweden, 2014 
The study of magnetic $M_{n+1} A X_{n}$ (MAX) phases ( $n=1-3, M$ - a transition metal, $A-$ an A group element, $X-\mathrm{C}$ or $\mathrm{N}$ ) is a recently established research area, fuelled by theoretical predictions and first confirmed experimentally through alloying of $\mathrm{Mn}$ into the well-known $\mathrm{Cr}_{2} \mathrm{AlC}$ and $\mathrm{Cr}_{2} \mathrm{GeC}$. Theoretical phase stability investigations suggested a new magnetic MAX phase, $\mathrm{Mn}_{2} \mathrm{GaC}$, containing $\mathrm{Ga}$ which is liquid close to room temperature. Hence, alternative routes for MAX phase synthesis were needed, motivating a further development of magnetron sputtering from liquid targets.

In this thesis, $\left(\mathrm{Cr}_{1-\mathrm{x}} \mathrm{Mn}_{\mathrm{x}}\right)_{2} \mathrm{GaC}(0 \leq x \leq 1)$ MAX phase thin films have been synthesized from elemental and/or compound targets, using ultra high vacuum magnetron sputtering. Initial thin film synthesis of $\mathrm{Cr}_{2} \mathrm{GaC}$ was performed using elemental targets, including liquid Ga. Process optimization ensured optimal target size and crucible geometry for containing the Ga. Films were deposited at $650{ }^{\circ} \mathrm{C}$ on $\mathrm{MgO}(111)$ substrates. X-ray diffraction and transmission electron microscopy confirms the growth of epitaxial $\mathrm{Cr}_{2} \mathrm{GaC}$ MAX phase with minor inclusions of $\mathrm{Cr}_{3} \mathrm{Ga}$.

To explore the magnetic characteristics upon $\mathrm{Mn}$ alloying, synthesis of $\left(\mathrm{Cr}_{0.5} \mathrm{Mn}_{0.5}\right)_{2} \mathrm{GaC}$ thin films was performed from elemental $\mathrm{Ga}$ and $\mathrm{C}$ and a composite $\mathrm{Cr} / \mathrm{Mn}$ target of 1:1 composition. Films were deposited on $\mathrm{MgO}(111), \mathrm{Al}_{2} \mathrm{O}_{3}(0001)$ (with or without $\mathrm{NbN}$ seed layer), and $4^{\circ}$ off-cut $4 \mathrm{H}-\mathrm{SiC}(0001)$ substrates. The films are smooth and of high structural quality as confirmed by X-ray diffraction and transmission electron microscopy. The film composition measured by high resolution energy dispersive X-ray spectroscopy confirms a composition corresponding to $\left(\mathrm{Cr}_{0.5} \mathrm{Mn}_{0.5}\right)_{2} \mathrm{GaC}$. The magnetic response, as measured with vibrating sample magnetometry, displays a ferromagnetic component, however, the temperature dependence of the magnetic moments and saturation fields suggests competing magnetic interaction and possible non-collinear magnetic ordering. 
Finally, inspired by theoretical predictions, a new member of the MAX phase family, $\mathrm{Mn}_{2} \mathrm{GaC}$, was synthesized. This is the first MAX phase containing $\mathrm{Mn}$ as a sole $M$ element. $\mathrm{X}$-ray diffraction and transmission electron microscopy confirms the characteristic MAX phase structure with a 2:1:1 composition. Theoretical work suggests that the magnetic ground state is almost degenerate between ferromagnetic and anti-ferromagnetic. Vibrating sample magnetometry shows ferromagnetic response with a transition temperature $T_{c}$ of $230 \mathrm{~K}$. However, also for this phase, complex magnetism is suggested. Altogether, the results indicate a new family of magnetic nanolaminates with a rich variation of magnetic ground states. 
This Licentiate thesis is part of my Ph.D. studies in the Materials Design group, Thin Film Physics division, at the Department of Physics, Chemistry, and Biology (IFM), at Linköping University, Sweden. It is a summary of my work between August 2011 and August 2014. The aim of my research is to develop methods for synthesis of novel MAX phases from elemental targets, including liquid $\mathrm{Ga}$. The methods are used for fundamental research on new MAX phases, with a particular focus on magnetic properties. The work has been performed in cooperation with University of Iceland (Iceland) and Uppsala University. Financial support has been provided by European Research Council (ERC). 



\section{Paper I}

Phase stability of $\mathrm{Cr}_{n+1} \mathrm{GaC}_{n} \mathrm{MAX}$ phases from first principles and $\mathrm{Cr}_{2} \mathrm{GaC}$ thin-film synthesis using magnetron sputtering from elemental targets

A. Petruhins, A. S. Ingason, M. Dahlqvist, A. Mockute, M. Junaid, J. Birch, J. Lu, L. Hultman, P. O. Å. Persson, and J. Rosen

Physica Status Solidi Rapid Research Letters 7, No. 11, 971-974 (2013)

\section{Paper II}

Synthesis and characterization of magnetic $\left(\mathrm{Cr}_{0.5} \mathrm{Mn}_{0.5}\right)_{2} \mathrm{GaC}$ thin films

A. Petruhins, A. S. Ingason, J. Lu, F. Magnus, S. Olafsson, and J. Rosen

Manuscript in final preparation

\section{Paper III}

A nanolaminated magnetic phase: $\mathrm{Mn}_{2} \mathrm{GaC}$

A. S. Ingason, A. Petruhins, M. Dahlqvist, F. Magnus, A. Mockute, B. Alling, L. Hultman, I. A. Abrikosov, P.O.Å. Persson and J. Rosen

Materials Research Letters, 2:2, 89-93 (2014) 



\section{ACKNOWLEDGEMENTS}

I would like to thank all the people, who have helped me to achieve all this, especially:

My supervisor Johanna Rosén

My co-supervisor Per Persson

My colleagues and co-authors:

Árni Sigurður Ingason

Aurelija Mockutė

Martin Dahlqvist

Friðrik Magnus

Jun Lu

Rahele Meshkian

Chung-Chuan Lai

And my loving wife Līga for providing support and motivation. 



\section{CONTENTS}

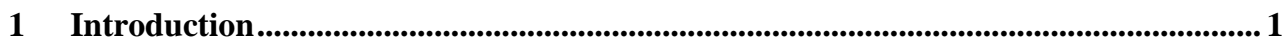

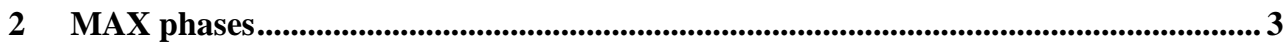

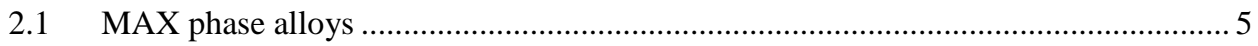

2.2 Mn incorporation in MAX phase materials .......................................................... 5

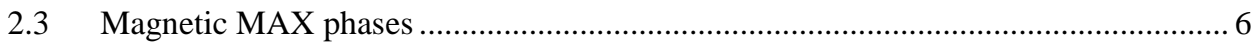

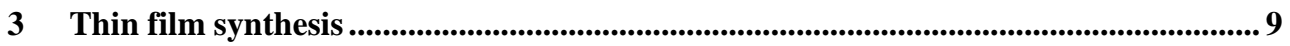

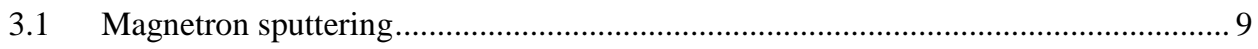

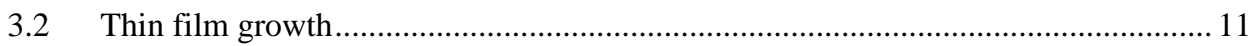

4 Thin film characterization .............................................................................................................. 13

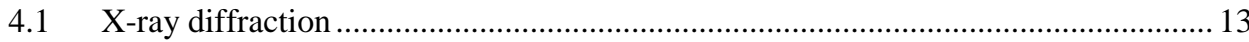

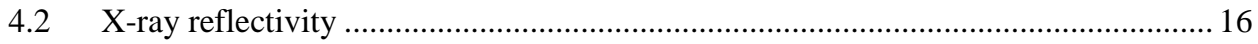

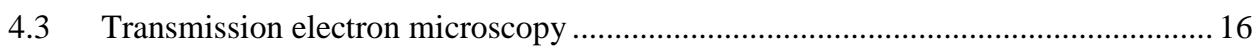

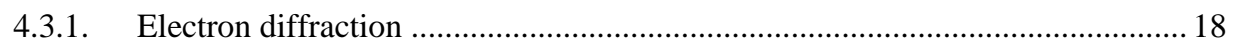

4.3.2. Energy dispersive X-ray spectroscopy (EDX) ................................................. 18

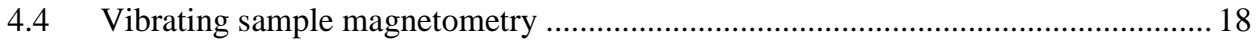

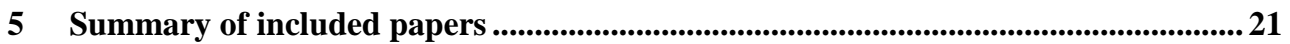

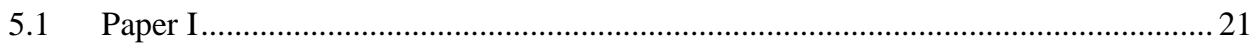

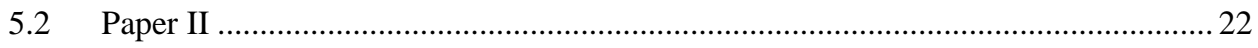

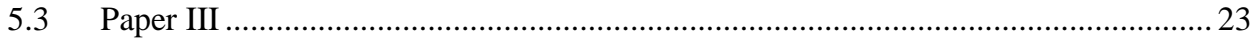

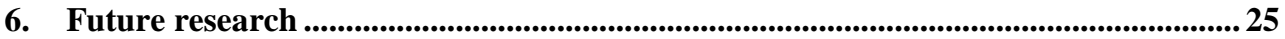

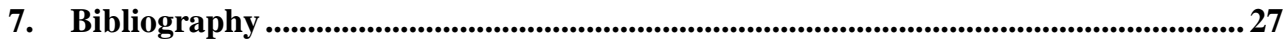





\section{INTRODUCTION}

Materials science is an interdisciplinary field comprising studies of materials and their properties, as well as discovery and design of new materials. Materials studies involve characterization of structure, properties and performance, thus allowing feedback to the synthesis process aiming for specific tailor-made materials. This scientific field is driven by an increasing demand on the materials' properties and performance, originating from a rapid development of new technologies. Implementation of these improved materials and processes by the industry creates added value for the society and the economy.

Thin films are encountered almost everywhere in the modern society. They are found as metal coating on mirrors, anti-reflective coatings on eyewear, and as scratch-resistant protective coating on your smartphone screen. Thin films are used to change or add properties of materials, for example, as a protective layer in hard coatings, or as an active part of a device, such as ferromagnetic coatings in a hard disk drive.

Magnetic materials have been known to humanity for a long time, and were used in compasses as early as $1000 \mathrm{BC}[1]$. Since then, the theory and understanding of magnetism has developed, and magnetic materials have found their way into numerous applications such as motors, hard disk drives, and medical imaging. However, technological advancements put demands on developing new and improved magnetic materials.

Layered magnetic materials have gained a lot of attention in the last decade. In 2007, the Nobel Prize in Physics was awarded to Albert Fert and Peter Grünberg for the discovery of giant magnetoresistance (GMR) in magnetic multilayers, a phenomenon that can be used for data storage and magnetic recording. As GMR is observed in multilayer structures, the applicability of different magnetic materials depends not only on magnetic properties, but also on structural quality, layer thickness, as well as interface quality. So called MAX phases all share the same archetypical structure with very similar in-plane lattice parameters, and therefore these materials could potentially be suitable for GMR applications, if endowed with suitable magnetic properties. 
MAX phases is a group of nanolaminated ternary carbides and nitrides, which chemical composition can be expressed with a general formula $M_{n+1} A X_{n}(n=1-3)$, where $M$ is a transition metal, $A$ - an A-group element, and $X$ is carbon or nitrogen [2,3]. They are inherently nanolaminated, suggesting anisotropic properties [4]. The characteristic layered structure for $M_{2} A X(n=1)$ is shown in Figure 1.

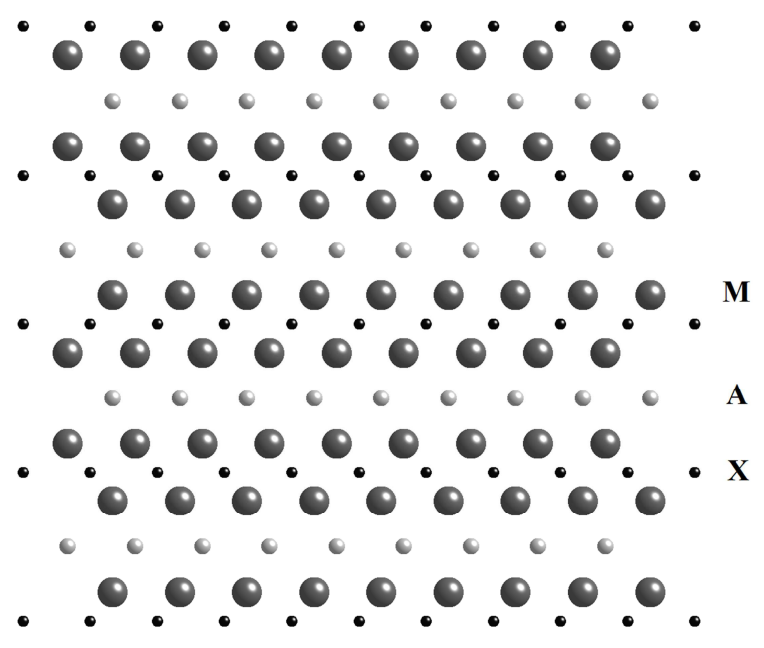

Figure 1. Nanolaminated structure of $M_{2} A X$ with $M_{2} X$ interleaved by layers of $A$ atoms.

Although many MAX phases have been synthesized in thin film form, most commonly by a method called magnetron sputtering, not all the MAX phase elements are straightforward to deposit in an ultrahigh vacuum (UHV) deposition system. For example, Ga has a melting point of $30^{\circ} \mathrm{C}$, and thus it will become liquid during deposition. This introduces several challenges for a well-controlled thin film synthesis process. However, Ga is a highly interesting element for development of novel magnetic MAX phases, as predicted by theoretical phase stability calculations.

The main objective of this thesis is to demonstrate use of $\mathrm{Ga}$ as an elemental target in a UHV sputtering system for deposition of novel MAX phase thin films. The method development opens a new, previously unexplored, area of thin film MAX phase materials. With a particular focus on high quality epitaxial thin film growth, vital for reliable material property investigations, attainable magnetic properties are explored. 


\section{MAX PHASES}

MAX phases were first discovered in the 1960's, though at the time known as Hphases, about 40 of them were synthesized alongside other carbides and nitrides [5]. However, it was not until the 1990's that scientific interest in these materials was reignited after demonstrating the unique combination of metallic and ceramic properties in a phase pure bulk sample of $\mathrm{Ti}_{3} \mathrm{SiC}_{2}$ [6]. Today, more than $60 \mathrm{MAX}$ phases exist, and Figure 2 shows the elements in the periodic table that form the $M_{n+1} A X_{n}$ phases known to date.

In 2002, $\mathrm{Ti}_{3} \mathrm{SiC}_{2}$ was synthesized as an epitaxial thin film for the first time [7]. Thin film growth of MAX phases allows highly oriented crystalline material for detailed characterization of properties. Since then, many MAX phases previously known as bulk material only have been synthesized also in thin film form, in addition to novel MAX phases which to this date have been reported only as thin films [8-10].

In the last decade, the MAX phase research field has evolved towards graphene-like 2D structures derived from MAX phases, the so called MXenes [11], as well as towards more in-depth studies of MAX phase properties such as mechanical [12], electrical [13], tribological [14], optical [15] and more recently magnetic properties [16-18].

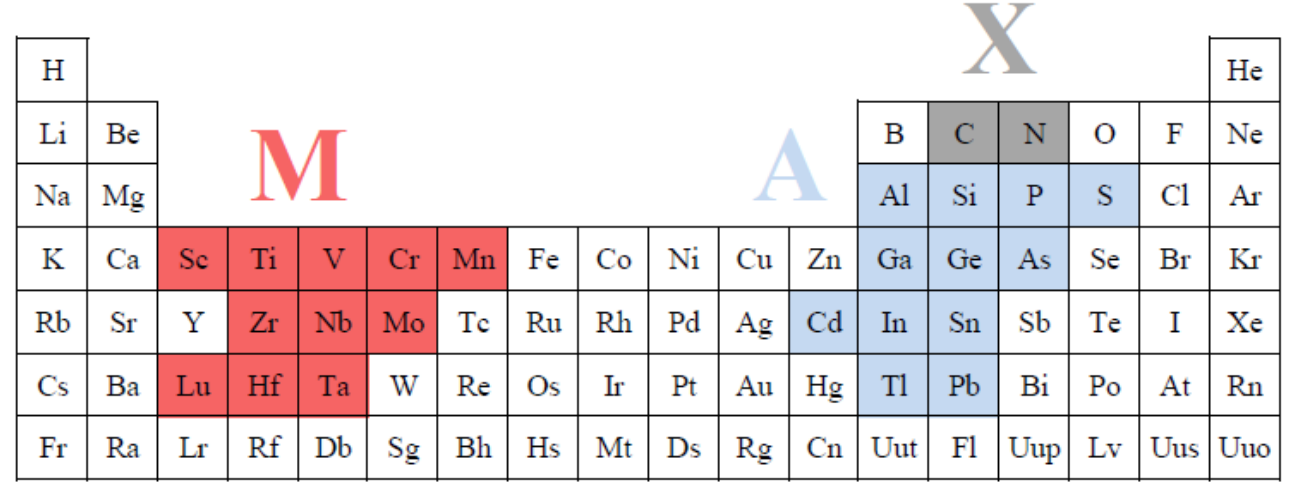

Figure 2. Periodic table with the $M, A$, and $X$ elements forming all $M_{n+1} A X_{n}$ phases known to date. 
All MAX phases share a hexagonal structure (space group $\mathrm{P}_{3} / \mathrm{mmc}$ ), in which edge sharing $M_{6} X$ octahedra are interleaved by layers of the $A$ element, hence forming a naturally nanolaminated material. Different stoichiometries of $M_{2} A X, M_{3} A X_{2}$ and $M_{4} A X_{3}$ are referred to as 211, 312 and 413, respectively. Unit cells of 211, 312, and 413 MAX phases can be seen in Figure 3. More complex stoichiometries have been observed, such as 514 phase [19], 615 [20], and 716 [21], as well as hybrid intergrown stoichiometries of 523 [8], interpreted as alternating layers of 211 and 312, and 725 [8], interpreted as alternating layers of 413 and 312 . These complex stoichiometries are, however, not regarded as members of the MAX phase family, since they are observed merely as minute grains or short stacking sequences within a more common MAX phase structure.

Since all MAX phases have the same structure and similar stoichiometries, similarities in their properties are also observed, such as high temperature oxidation resistance and machinability [3], good electrical and thermal conductivity [6] as well as excellent damage and thermal shock tolerance [22].
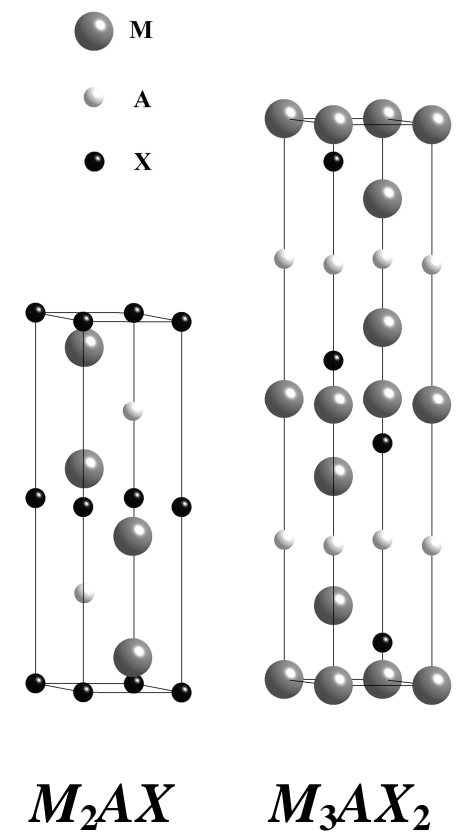
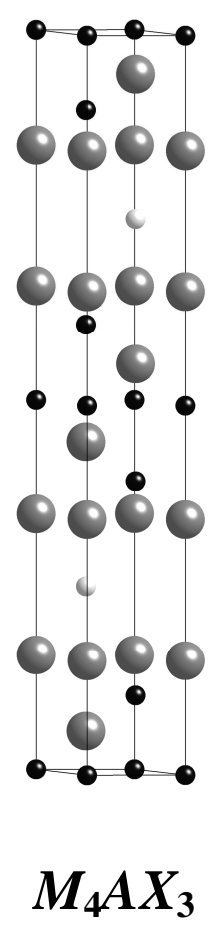

Figure 3. Unit cells of $M_{2} A X(211), M_{3} A X_{2}$ (312) and $M_{4} A X_{3}$ (413) phases. 


\subsection{MAX phase alloys}

As MAX phases are formed from neighbouring elements in the periodic table, there is a great opportunity for alloying into isostructural solid solutions on $M, A$ and $X$ sites. Such alloys have been extensively studied, e.g., ( $\mathrm{Nb}, \mathrm{V})_{2} \mathrm{AlC}[23], \mathrm{Cr}_{2}(\mathrm{Al}, \mathrm{Ge}) \mathrm{C}$ [24], and $\mathrm{Ti}_{2} \mathrm{Al}(\mathrm{C}, \mathrm{N})$ [25]. Alloying on $M$ and $A$ sites has been most studied due to a large number of attainable elemental combinations, while studies on $X$ site alloying are limited due to the two elements only available. However, experimental observations have suggested a significant amount of oxygen substitution on the $X$ site while retaining the MAX phase structure [26,27]. These observations were later supported by ab-initio calculations [4,28] indicating possible tuning of the material properties with change in oxygen content. Oxygen incorporation as interstitials was later also studied both theoretically and experimentally for $\mathrm{Cr}_{2} \mathrm{AlC}$ [29].

Substitution of one element with another gives the opportunity to tune the material properties, by adjusting the amount of the element substituted. The material properties in solid solutions can change in monotonous manner, e.g. the thermal expansion of $\mathrm{Cr}_{2} \mathrm{AlC}$ changes linearly when substituting $\mathrm{Al}$ with Ge throughout the whole composition range of $\mathrm{Cr}_{2}(\mathrm{Al}, \mathrm{Ge}) \mathrm{C}$ [24]. However, alloying can also give rise to properties that are superior compared to the pure MAX phase constituents, for example, the $\mathrm{Ti}_{2} \mathrm{AlC}_{0.5} \mathrm{~N}_{0.5}$ solid solution exhibits approx. $20 \%$ higher hardness than $\mathrm{Ti}_{2} \mathrm{AlC}$ and almost $30 \%$ higher than $\mathrm{Ti}_{2} \mathrm{AlN}$ [25].

\subsection{Mn incorporation in MAX phase materials}

Although Mn is marked as an element belonging to the MAX phase family in Figure 2, it was introduced only very recently. In 2010, Dahlqvist et al. developed a method for theoretically predicting the phase stability of MAX phases. The method was benchmarked and could completely reproduce experimental occurrences of a large set of known stable MAX phases [30]. Later, the same method was used for predicting the previously unknown $\mathrm{Nb}_{2} \mathrm{GeC}$ MAX phase, which was subsequently synthesized, and hence could demonstrate the predictive power of the method [31]. This result motivated a search for new MAX phases composed of new MAX phase elements. An initial study was performed on the stability of alloys with $\mathrm{Mn}$ on the $M$ site, and a stable $\left(\mathrm{Cr}_{1-\mathrm{x}} \mathrm{Mn}_{\mathrm{x}}\right)_{2} \mathrm{AlC} \mathrm{MAX}$ phase alloy was predicted [32], and thereafter synthesized [17]. Later also ( $\mathrm{Cr}, \mathrm{Mn})_{2} \mathrm{GeC}$ solid solutions were identified as theoretically stable, and successful synthesis of $\left(\mathrm{Cr}_{0.75} \mathrm{Mn}_{0.25}\right)_{2} \mathrm{GeC}$ thin films were thereafter reported [16]. 
$(\mathrm{Cr}, \mathrm{Mn})_{2} \mathrm{AlC}$ and $(\mathrm{Cr}, \mathrm{Mn})_{2} \mathrm{GaC}$ solid solutions have also been studied in bulk form, and the results of this study suggest that the solubility of $\mathrm{Mn}$ in bulk $\mathrm{Cr}_{2} \mathrm{AlC}$ and $\mathrm{Cr}_{2} \mathrm{GaC}$ MAX phases is likely lower than in thin films [33]. Therefore, in the case of bulk synthesis, the ratios of the initial powder constituents cannot be used for stating the MAX phase composition. Similar results were also obtained for $\left(\mathrm{Cr}_{1-\mathrm{x}} \mathrm{Mn}_{\mathrm{x}}\right)_{2} \mathrm{GeC}$ solid solutions, where already at $x=0.1$ a noticeable amount of Mn containing impurity phases appeared, once again indicating the possibly of a lower solubility limit of $\mathrm{Mn}$ in the bulk material [34]. Claims of $\left(\mathrm{Cr}_{1-\mathrm{x}} \mathrm{Mn}_{\mathrm{x}}\right)_{2} \mathrm{GaC}$ solid solutions with $\mathrm{x}$ up to 0.5 have been reported in bulk [35], however no compositional analysis was provided in the study.

\subsection{Magnetic MAX phases}

MAX phases containing Mn have attracted a lot of attention in the research community by introducing a new property previously not associated with the MAX phase family - magnetism.

The initial search for a magnetic MAX phase did not include Mn, but rather focused on the more commonly recognized ferromagnetic (FM) element Fe. The hypothetical magnetic $\mathrm{Fe}_{\mathrm{n}+1} A \mathrm{C}_{n}(n=1-3, A=\mathrm{Al}$, Si or Ge) were theoretically investigated by Luo et al. [36], and the study suggested that $\mathrm{Fe}_{3} \mathrm{AlC}_{2}$ was stable and ferromagnetic with a magnetic moment of $0.73 \mu_{\mathrm{B}}$ per $\mathrm{Fe}$ atom. However, no Fe-Al-C MAX phases have been found experimentally.

In a later study by Dahlqvist et al. the $\mathrm{Fe}_{3} \mathrm{AlC}_{2} \mathrm{MAX}$ phase was found not stable, along with the other MAX phases in the Fe-Al-C system [32]. This work explored the phase stability of $M_{n+1} \mathrm{AlC}_{\mathrm{n}}(n=1-3$ and $M=\mathrm{Cr}, \mathrm{Mn}, \mathrm{Fe}, \mathrm{Co})$. It was found that magnetic MAX phases based on $M=\mathrm{Mn}, \mathrm{Fe}$ and $\mathrm{Co}$ are unstable, however, $\left(\mathrm{Cr}_{1-\mathrm{x}} \mathrm{Mn}_{\mathrm{x}}\right)_{2} \mathrm{AlC}$ alloys were not only predicted to be stable at least up to $x=0.5$, they were also suggested to be ferromagnetically ordered. An experimental study on $\left(\mathrm{Cr}_{0.8} \mathrm{Mn}_{0.2}\right)_{2} \mathrm{AlC}$ by Mockute et al. revealed that corresponding thin films show magnetic response, with indication of a transition temperature well above room temperature [18]. $\left(\mathrm{Cr}_{0.75} \mathrm{Mn}_{0.25}\right)_{2} \mathrm{GeC}$ thin films also showed magnetic response up to the maximum measurement temperature of $300 \mathrm{~K}$ [16]. At a temperature of $50 \mathrm{~K}$ the films had a saturation magnetic moment of $\mathrm{m}_{\mathrm{s}}=0.36 \mu_{\mathrm{B}}$ per $\mathrm{Mn}$ atom, with remanent moment $\mathrm{m}_{\mathrm{r}}=0.031 \mu_{\mathrm{B}}$. These numbers are smaller than those predicted from theory for $\mathrm{FM}$ ordering $\left(\mathrm{m}_{\mathrm{s}} \approx 2 \mu_{\mathrm{B}}\right)$, which could indicate competing magnetic interactions. 
This is not surprising as $\mathrm{Mn}$ is known to display complex magnetic ordering, like chiral magnetic ordering in Mn monolayers [37].

The report on bulk $\left(\mathrm{Cr}_{1-\mathrm{x}} \mathrm{Mn}_{\mathrm{x}}\right)_{2} \mathrm{AlC}$ and $\left(\mathrm{Cr}_{1-\mathrm{x}} \mathrm{Mn}_{\mathrm{x}}\right)_{2} \mathrm{GaC}$ [33] showed that the former did not exhibit magnetic response for a Mn content of only $x=0.06$ whereas the latter had a Mn content of $x=0.3$, revealing two magnetic transitions at $T_{t_{1}}=38 \mathrm{~K}$ and $T_{t_{2}}=153 \mathrm{~K}$. Another study on $\left(\mathrm{Cr}_{1-\mathrm{x}} \mathrm{Mn}_{\mathrm{x}}\right)_{2} \mathrm{GaC}$ solid solutions with allegedly $x=0.5$, showed paramagnetic response with a weak ferrimagnetism at the ground state [35]. Furthermore, a report on bulk $\left(\mathrm{Cr}_{1-\mathrm{x}} \mathrm{Mn}_{\mathrm{x}}\right)_{2} \mathrm{GeC}$ solid solutions suggested the coexistence of ferromagnetic and re-entrant cluster glass state in the material [34]. Segregation of Mn into Mn-poor and Mnrich regions was also suggested in the study. Altogether, the field of magnetic MAX phases is very young, and further understanding and a more comprehensive picture of the detailed magnetic properties is required for the materials discovered to date.

In this thesis, growth of Ga containing MAX phases has been demonstrated, enabling the discovery of a new MAX phase, $\mathrm{Mn}_{2} \mathrm{GaC}$, and in turn adding a new element, $\mathrm{Mn}$, to the MAX phase family. 



\section{THIN FILM SYNTHESIS}

Growth of thin films from vapour phase can be divided into two main groups: physical vapour deposition (PVD) and chemical vapour deposition (CVD). PVD employs purely physical processes for generation of vapour which is condensed on a substrate, for example thermal evaporation or magnetron sputtering. In CVD the growth occurs through chemical reactions of species forming the desired material. The latter process takes place at thermodynamical equilibrium and thus requires high temperatures. In this thesis, only magnetron sputtering has been used for thin film synthesis.

\subsection{Magnetron sputtering}

Sputtering is a process where ion bombardment of the target material causes ejection of atoms. An inert gas, typically Ar, is introduced into a vacuum chamber, and is ionized through collisions with other Ar atoms or secondary electrons. By applying a negative bias to the target (the material to be deposited), $\mathrm{Ar}^{+}$ions are accelerated towards the target, colliding with the target material from which atoms are ejected (sputtered). The energy of these atoms is typically on the order of a few $\mathrm{eV}$, and they are then transported to the substrate where they are deposited to form a film.

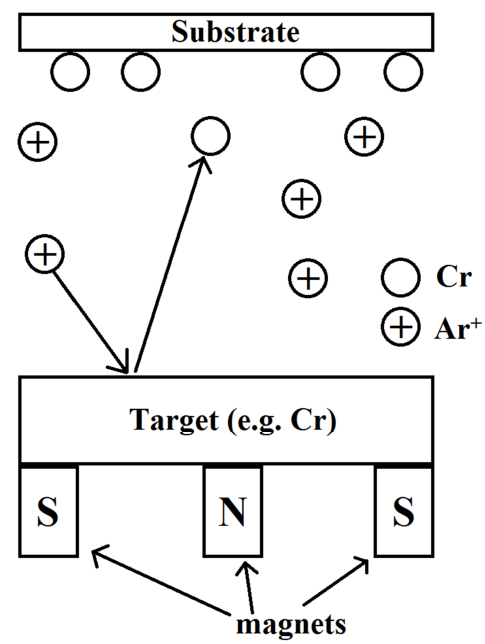

Figure 4. Basic schematic drawing of the magnetron sputtering process. 
In conventional sputtering, a high partial pressure of Ar is required for initiating a stable sputtering process. This is unfavourable due to a large amount of collisions of the target atoms on their way towards the substrate, where they lose energy and are scattered, which decreases the growth rate. Low energy of the species that arrive to the substrate can cause deterioration of the crystal quality of the film due to limited adatom mobility. To increase the ionization degree of Ar, magnets are placed behind the target, thus trapping electrons and increasing the ionization probability in the vicinity of the target. A higher ionization degree allows a reduction in the partial pressure of Ar, which in turn results in enhanced growth rates. In Figure 4, a schematic drawing of magnetron sputtering process is shown.

Sputtering of a compound material can be done in several ways, most commonly:

1) Using a compound target.

2) Using elemental targets and a reactive gas (reactive sputtering).

3) Using elemental targets that form the compound.

In the case of sputtering from a compound target, several problems can arise, e.g., differences in angular and energy distributions of the sputtered species and scattered atoms that result in films with different stoichiometry as compared to that of the target. In addition, deviations from target stoichiometry in the film can arise from different sticking probabilities of the elements. For example, this has been reported for attempted synthesis of $\mathrm{Ti}_{3} \mathrm{SiC}_{2}$ thin films sputtered from a compound $\mathrm{Ti}_{3} \mathrm{SiC}_{2}$ target [39].

In reactive sputtering, the film is formed from a chemical reaction between the constituent target atoms and the gas. The film stoichiometry can be controlled by the partial pressures of the inert and the reactive gas. This method is used for deposition of nitrides [40], oxides [41], hydrides [42], fluorides [42], sulphides [38], borides [43], carbides [40], etc., and the method can be used for deposition of nitride MAX phase thin films, e.g. Ti 2 AlN [44].

When applicable, the use of elemental targets is often a preferred method for deposition of compounds, because it provides a higher degree of freedom allowing control of each of the elements independently. It is often the choice for growth of carbide MAX phase thin films, e.g. $\mathrm{Ti}_{3} \mathrm{SiC}_{2}$ [7]. Sometimes, due to constraints in the design of the deposition system, the number of available targets is below the number of elements forming the compound. In this case, two of the elements can be used in a binary compound target. For the case of MAX phase carbides, three targets are necessary to ensure the full degree of freedom 
for each of the constituting elements. However, when depositing MAX phase alloys either a four target deposition system needs to be used [17] or a compound target [16], where the former allows a larger variation of the alloy composition.

\subsection{Thin film growth}

After being sputtered, the species in the vapour phase are transported to the substrate where they condense and form a film. Atoms that arrive on the substrate are called adatoms. Typical kinetic energies of adatoms in magnetron sputtering are few eV. Such energies can be sufficient for adatom diffusion on the surface, overcoming the Ehrlich-Schwoebel barrier to either find other adatoms, to form clusters or to be desorbed (evaporated). Formed clusters are not stable until they reach a critical nucleus size and continue to grow. By increasing the temperature, the number of these nucleation centres decrease, due to enhanced adatom mobility. The growth can be classified in three characteristic modes (Figure 5):

1. Frank-van der Merwe or layer-by-layer growth,

2. Stranski-Krastanov or layer-by-layer followed by island growth.

3. Volmer-Weber or island growth,

1.

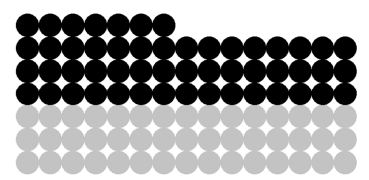

2.

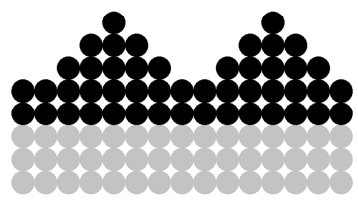

3.

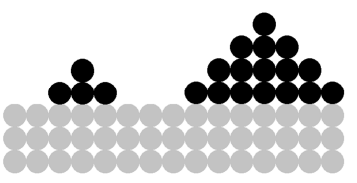

Figure 5. Thin film growth modes: 1. Layer-by-layer growth, 2. layer-by-layer followed by island growth, and 3. island growth.

Single crystals are often chosen as substrates, with specific atomic planes facing the plasma flux as incoming adatoms tend to diffuse to specific crystallographic sites on the substrate surface to minimizing the interfacial free energy. Such growth is called epitaxial growth. To achieve epitaxial growth of $(000 \ell)$ oriented MAX phases, single crystals of $\mathrm{Al}_{2} \mathrm{O}_{3}(0001), \quad \mathrm{SiC}(0001)$, and $\mathrm{MgO}(111)$ are most commonly used. 



\section{THIN FILM CHARACTERIZATION}

\subsection{X-ray diffraction}

$\mathrm{X}$-ray diffraction is a simple yet very powerful non-destructive tool for identification of different crystalline phases in the film, as well as crystal texture, crystal quality, grain size, stress, etc.

X-rays are electromagnetic radiation with wavelength ranging from 0.1 to $50 \AA$, which is on the same order as atomic separation distances in crystals. As atoms are arranged in a periodic manner in a crystal, irradiating such sample with X-rays results in constructive interference of the scattered X-rays from the periodic lattice when Bragg's law is fulfilled:

$$
2 d \sin \theta=n \lambda
$$

where $\mathrm{d}$ is the spacing between atomic planes, $\theta$ is the scattering angle, $n$ is an integer, and $\lambda$ is the wavelength, see Figure 6.

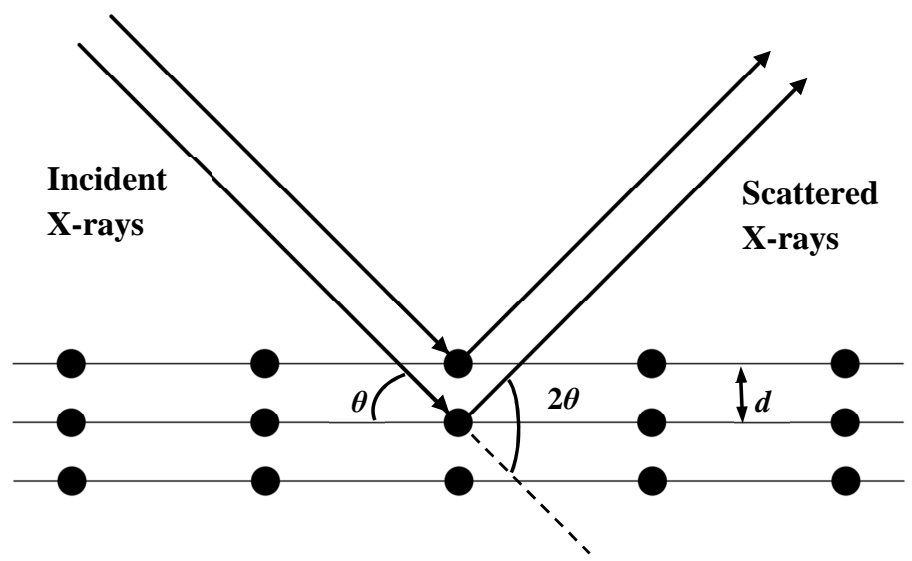

Figure 6. Simplified schematic drawing of X-ray diffraction.

Bragg's law is the minimum requirement for diffraction. Condition for constructive interference is also determined by the structure factor of the crystal. The combination of 
crystal structure and composition is what uniquely defines a material and makes identification of different phases possible. The combination determines the diffractogram of a crystalline material, which is the characteristic set of diffraction angles and diffracted beam intensities.

Widely used are measurements in $\theta-2 \theta$ geometry, where the detection angle is the same as the incidence angle. Both angles are changed simultaneously, thus it is a symmetric scan. In this geometry, only lattice planes parallel to the surface are probed. For polycrystalline samples, e.g., powder, the planes are oriented randomly and are present in all directions in the sample. Therefore, there will always be planes that are parallel to the surface and all allowed reflections can be detected. In the case of thin films, however, some crystal growth directions may be more favourable than others, resulting in a textured film, meaning that only the peaks from certain set of planes are present. For example, for a MAX phase

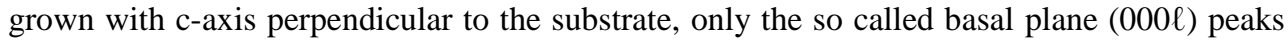
appear in the diffractogram, see Figure 7.

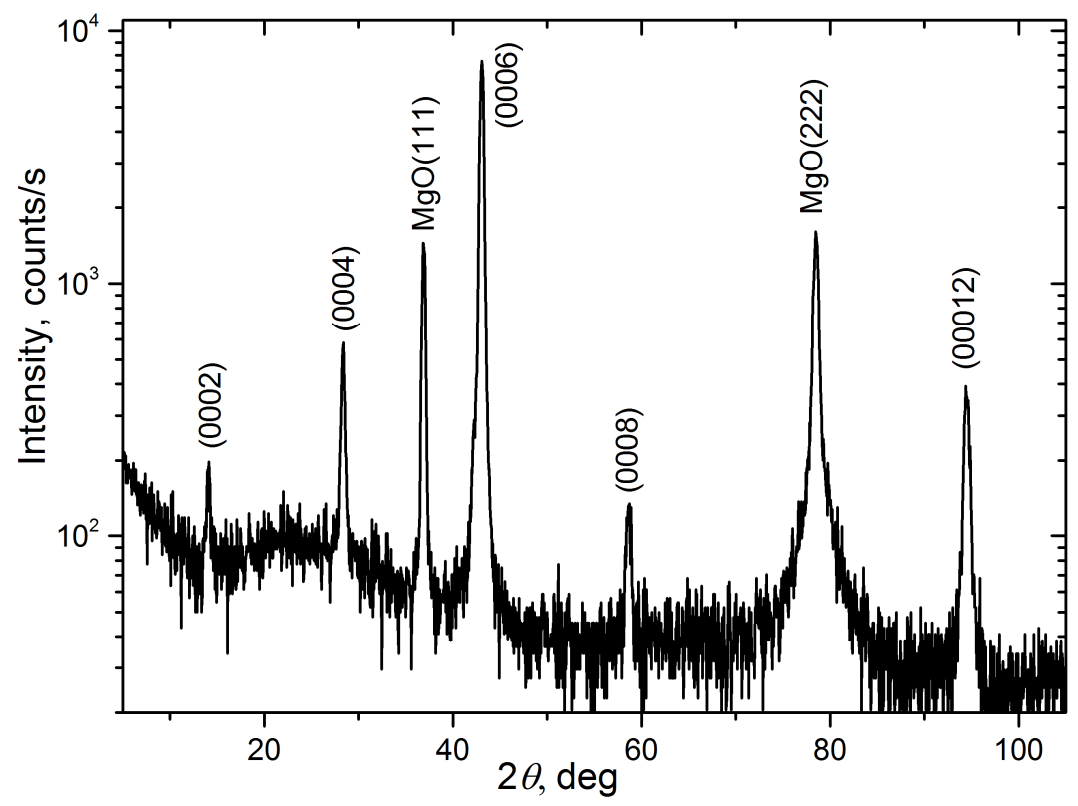

Figure 7. XRD $\theta / 2 \theta$ scan of a $\left(\mathrm{Cr}_{0.5} \mathrm{Mn}_{0.5}\right)_{2} \mathrm{GaC}$ thin film deposited on $\mathrm{MgO}(111)$ substrate. Only basal plane peaks from the MAX phase and substrate peaks are visible in the diffractogram.

As established before, in a symmetric $\theta-2 \theta$ geometry, the signal originates only from those atomic planes that are oriented parallel to the sample surface. This only accounts for a 
fraction of the crystalline planes of the phase. To obtain information about different orientations in the crystal, pole measurements are performed. In pole measurements, the probed $2 \theta$ angle is fixed and selected to match the desired atomic spacing. Then the sample is tilted along the azimuth $\chi$ and rotated along $\varphi$ (Figure 8). The data most commonly is presented in circular colour maps displaying intensity in polar coordinates $I(\chi, \varphi)$. Randomly oriented samples will show uniformly random distributed intensity at all $\chi$ and $\varphi$ angles. Textured films, where grains are oriented in the same way out-of-plane but randomly in-plane will show diffraction at certain $\chi$ values and for all $\varphi$ values, displayed as circles in pole figure. However, for the films that are epitaxial, i.e. grains are oriented in a way determined by the substrate both out-of-plane and in-plane, diffraction occurs only at certain values of $\chi$ and $\varphi$, which is seen as spots in pole figures. The number of spots and distance between them is determined by the symmetry of the crystal plane that is analysed. The $\chi$ is then the angle between the plane that is analysed and the planes that are parallel to the surface. These measurements help to determine the epitaxial relationship between the substrate and the film as well as are a very powerful tool to confirm a crystal structure of an unknown phase in the film.

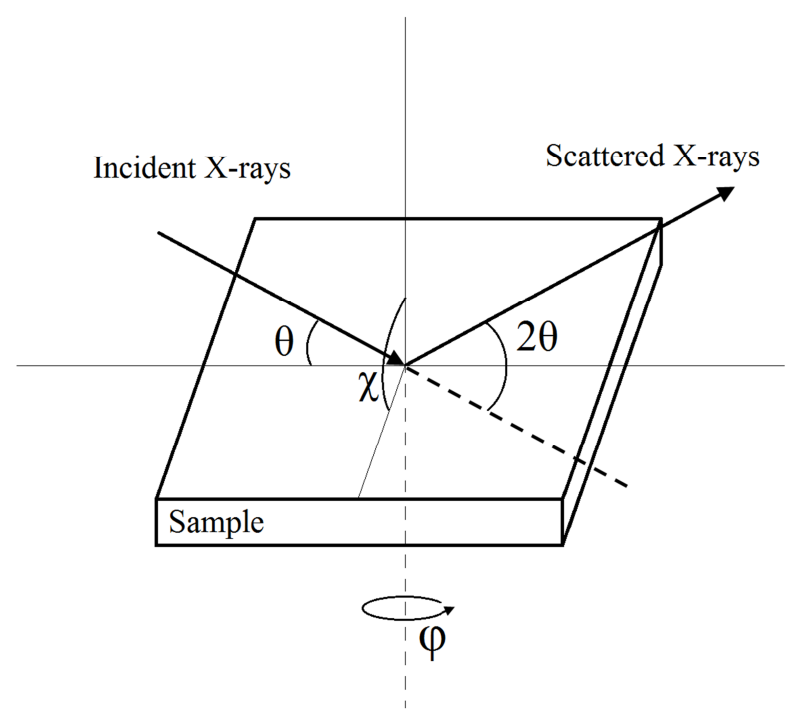

Figure 8. Schematic drawing of a pole measurement. 


\subsection{X-ray reflectivity}

$\mathrm{X}$-ray reflectivity (XRR) is a method based on the symmetric $\theta-2 \theta$ geometry at grazing angles, where the probed spacing becomes comparable to film thickness, and scattering occurs from film surface and interfaces. It is a useful method providing information such as thickness, density and roughness. XRR analysis can be performed on both crystalline and amorphous materials, however, the surfaces should be smooth and interfaces sharp, and the films should be thin, typically less than $100 \mathrm{~nm}$.

\subsection{Transmission electron microscopy}

Transmission electron microscopy (TEM) is a very useful analysis technique for investigating samples on atomically resolved scale. The essence of TEM is very similar to that of an optical microscope. It is known that the resolution of the optical microscope is limited by the wavelength of incident light $(\sim 500 \mathrm{~nm})$, and therefore, for improved resolution radiation with shorter wavelength must be used. Concluded from the wave-particle duality, electrons accelerated to $200 \mathrm{keV}$ have a resulting wavelength of $2.5 \mathrm{pm}$. This electron beam is focused with electromagnetic lenses, and the point resolution achieved is usually $\sim 1 \AA$, which enables atomically resolved analysis.

Thin film TEM sample preparation is destructive to part of the sample. Ideally, the samples should be to be very thin $(<100 \mathrm{~nm}$, depends on the acceleration voltage used) over a large area, contain no artefacts from the preparation process, be clean from contaminants, be fairly conductive and non-magnetic, as well as able to represent the whole sample.

As the electron beam irradiates the sample, the beam is not only transmitted but also scattered, absorbed and diffracted. Other types of signals are generated after interaction with the sample, such as X-rays, secondary and Auger electrons. This makes interpretation of TEM results non-trivial.

Contrast in TEM can be generated in several ways. Diffraction contrast is formed when a coherent electron beam is scattered elastically after being irradiated on the sample according to Bragg's law. A diffraction pattern is created in the form of spots, where the centre spot corresponds to the incident beam and the other spots correspond to a certain set of atomic planes. Images can be generated from these spots or set of spots by inserting an objective aperture in the diffraction plane. In bright field, only the transmitted beam is 
allowed to pass through the apertures while other spots are blocked. In dark field, the transmitted beam is blocked but other spots are allowed to pass through. This aperture can help to investigate different features in the sample, e.g. dislocations, strain etc.

For high resolution TEM (HR-TEM) imaging, phase contrast is used. As an electron wave goes through the sample, it interacts with the crystal potential and experiences a phase shift. The phase is also shifted by the microscope, which is conventionally defined by the contrast transfer function (CTF). For conventional (not aberration corrected) electron microscopes, the contrast function can exhibit contrast reversal and oscillations, making it challenging to interpret the contrast in HR-TEM images. An example of a HR-TEM image is shown in Figure 9.

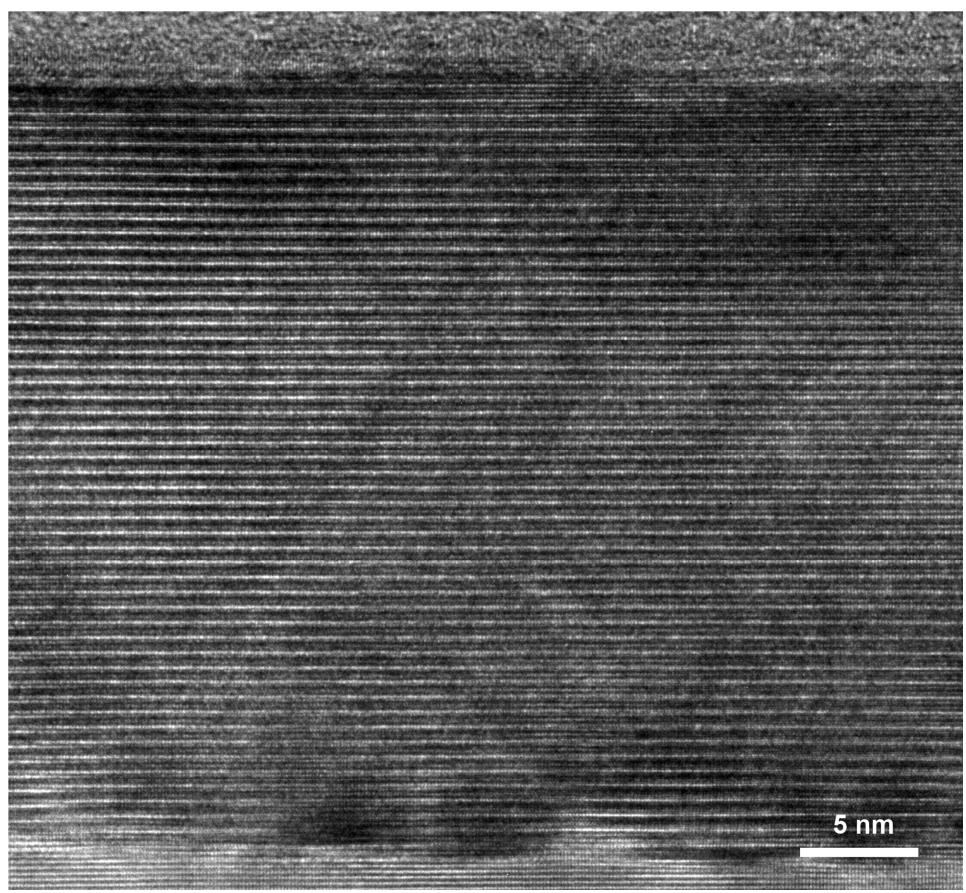

Figure 9. HR-TEM image of $\left(\mathrm{Cr}_{0.5} \mathrm{Mn}_{0.5}\right)_{2} \mathrm{GaC}$ MAX phase (middle) and $\mathrm{MgO}$ substrate (bottom)

Scanning transmission electron microscopy (STEM) is an operation mode in TEM where an electron beam is focused into a fine electron probe which is scanning the sample. Each pixel on the resulting image is generated by the scattered intensity at that point. The resolution is thus determined by the size of the electron probe. Typically, these images are 
recorded by the high-angle annular dark-field (HAADF) detector. In this setup, the detected intensity $(I)$ is proportional on the square of atomic number $(Z)$ and specimen thickness $(t)$ : $I \sim Z^{2} t$. Images formed by this contrast are relatively easy to interpret and allows imaging of sample regions that are too thick to be imaged with conventional TEM.

\subsubsection{Electron diffraction}

A diffraction pattern is formed together with the TEM image, and the setup can therefore be set so that the diffraction plane is projected on the screen or recording device, rather than the image. It provides information about the crystal structure, lattice spacing and orientation. Also, an aperture can be inserted in the image plane, allowing a diffraction pattern obtained from a specific region of the sample. This can be used to obtain information from single grain in a sample containing different phases.

\subsubsection{Energy dispersive X-ray spectroscopy (EDX)}

When irradiated by the electron beam, the electrons in the sample atoms are excited to higher levels, and when de-excitation to ground level occurs, photons (X-rays) or electrons (Auger electrons) are emitted. Since each atom has a unique atomic structure, there is also a set of unique peaks in X-ray spectrum, allowing elemental identification and bonding information.

\subsection{Vibrating sample magnetometry}

Vibrating sample magnetometry (VSM) is a method that measures the magnetic properties of the sample. It was developed in 1955 by Simon Foner [45]. A sample is placed in a uniform magnetic field and is magnetized, aligning the spins along the field direction, see Figure 10. The sample is then vibrated sinusoidally, inducing a current in surrounding pickup coils which is proportional to the magnetic moment in the sample, but not dependent on the strength of applied field. 


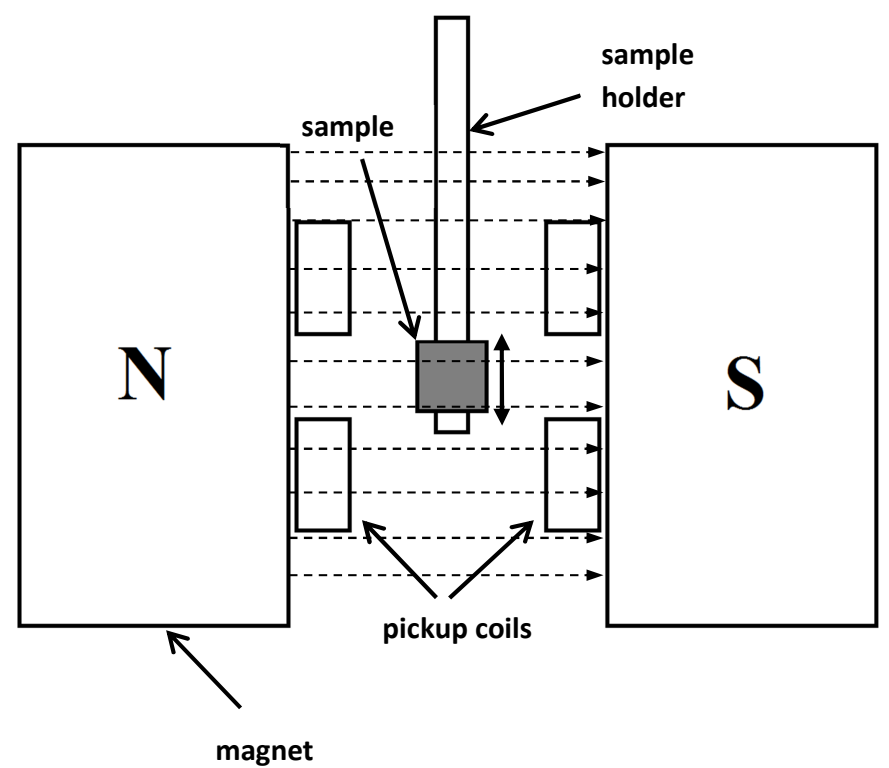

Figure 10. Schematic of a VSM measurement setup. 



\section{SUMMARY OF INCLUDED PAPERS}

\subsection{Paper I}

\section{Phase stability of $\mathrm{Cr}_{n+1} \mathrm{GaC}_{n} \mathrm{MAX}$ phases from first principles and $\mathrm{Cr}_{2} \mathrm{GaC}$ thin-film synthesis using magnetron sputtering from elemental targets}

In this paper, a theoretical study on the stability of $\mathrm{Cr}_{n+1} \mathrm{GaC}_{n}(n=1-3) \mathrm{MAX}$ phases is reported. The previously developed method for investigating phase stability was used [30], including evaluation of several different magnetic spin configurations. The latter has been shown to significantly affect the predicted material's properties, e.g., bulk modulus [46], also compared to non-magnetic configurations. The calculations show that out of investigated $\mathrm{Cr}_{n+1} \mathrm{GaC}_{n}(n=1-3)$, only the $\mathrm{Cr}_{2} \mathrm{GaC}$ MAX phase is stable, consistent with previous experimental observations.

Magnetron sputtering of $\mathrm{Cr}, \mathrm{Ga}$, and $\mathrm{C}$ was performed from elemental targets onto $\mathrm{MgO}(111)$ substrates at $650{ }^{\circ} \mathrm{C}$. XRD results show thin films mainly consisting of epitaxial $\mathrm{Cr}_{2} \mathrm{GaC}$ MAX phase with small amount of $\mathrm{Cr}_{3} \mathrm{Ga}$ impurity phase. Lattice parameters of $\mathrm{a}=2.89 \AA$ and $\mathrm{c}=12.62 \AA$ were obtained for the thin films, which are in good agreement with those from bulk synthesis ( $a=2.90 \AA$ and $c=12.63 \AA$ ). TEM analysis is consistent with the XRD results, showing primarily $\mathrm{Cr}_{2} \mathrm{GaC}$ MAX phase, though with few identified grains of $\mathrm{Cr}_{3} \mathrm{Ga}$.

This paper is the first report on a Ga-based MAX phase in thin film form, synthesized from elemental targets. The developed synthesis approach is generic, and can be used for synthesis of other multicomponent materials. Though, for MAX phases in particular, the results allow further exploration of new thin film MAX phases based on Ga. 


\subsection{Paper II}

\section{Synthesis and characterization of magnetic $\left(\mathrm{Cr}_{0.5} \mathrm{Mn}_{0.5}\right)_{2} \mathrm{GaC}$ thin films}

In this paper, $\left(\mathrm{Cr}_{1-\mathrm{x}} \mathrm{Mn}_{\mathrm{x}}\right)_{2} \mathrm{GaC}$ has been synthesized from a compound $\mathrm{Cr} / \mathrm{Mn}$ target of 1:1 composition together with elemental $\mathrm{Ga}$ and $\mathrm{C}$ targets. $\mathrm{MgO}, \mathrm{Al}_{2} \mathrm{O}_{3}$, and $\mathrm{SiC}$ substrates were used in order to identify optimal conditions for single phase epitaxial growth. XRD analysis reveals epitaxial MAX phase of high structural quality, with no trace of tilted grain growth for a $\mathrm{MgO}$ substrate, which is confirmed by TEM analysis. From EDX analysis a Mn content of 25 at. \% can be concluded, and thus a thin film composition of $\left(\mathrm{Cr}_{0.5} \mathrm{Mn}_{0.5}\right)_{2} \mathrm{GaC}$.

Magnetic properties were determined by VSM in the temperature range $30-300 \mathrm{~K}$. The sample shows ferromagnetic response in the whole range, with a magnetization at $5 \mathrm{~T}$ decreasing linearly with increasing temperature, with no indication of a distinct magnetic transition Isothermal magnetization vs. magnetic field shows a sharp increase in magnetization at low fields up to $0.2 \mathrm{~T}$, with further increase up to $5 \mathrm{~T}$, where no saturation is reached. This indicates competing magnetic interaction in the system.

These results show the effect of Mn incorporation on the suggested antiferromagnetic ground state of pure $\mathrm{Cr}_{2} \mathrm{GaC}$. The acquired high quality thin film with respect to both phase composition and structure ensures that the analysed magnetic properties originate from the MAX phase, and not defects or impurity phases. Evidently, Mn incorporation can be used to change the magnetic properties of the MAX phase to include a ferromagnetic component, and the suggested competing interactions indicate a variety of attainable magnetic configurations. 


\subsection{Paper III}

\section{A nanolaminated magnetic phase: $\mathrm{Mn}_{2} \mathrm{GaC}$}

In this paper, the phase stability and magnetic properties have been calculated from first principles for the hypothetical MAX phase $\mathrm{Mn}_{n+1} \mathrm{GaC}_{n}(n=1-3) . \mathrm{Mn}_{2} \mathrm{GaC}$ was identified as a stable phase. Several magnetic spin configurations were evaluated, suggesting a magnetic ground state being practically degenerate between ferromagnetic and antiferromagnetic ordering.

Thin film synthesis was performed using magnetron sputtering from elemental $\mathrm{Mn}, \mathrm{Ga}$ and $\mathrm{C}$ targets onto a $\mathrm{MgO}(111)$ substrate at $550{ }^{\circ} \mathrm{C}$. XRD $\theta / 2 \theta$ and pole measurements confirmed the characteristic MAX phase structure with lattice parameters of $a=2.90 \AA$ and $\mathrm{c}=12.55 \AA$ A. STEM analysis revealed a film consisting of large (>200 $\mathrm{nm})$ MAX phase grains, and EDX elemental mapping performed on these grains reveal a homogenous composition with a relative content of 52 at. $\% \mathrm{Mn}, 23$ at. $\% \mathrm{Ga}$, and 23 at. \% C.

VSM results show that the MAX phase exhibits ferromagnetic response at temperatures up to and including $230 \mathrm{~K}$, with a clearly displayed hysteresis in the magnetization curves. At $50 \mathrm{~K}$, the magnetic moment $\mathrm{m}_{\mathrm{s}}$ at $5 \mathrm{~T}$ was found to be $0.29 \mu_{\mathrm{B}}$ (per $\mathrm{Mn}$ atom) with a remanent moment $\mathrm{m}_{\mathrm{r}}$ of $0.15 \mu_{B}$ (per Mn atom). The coercive field was $9 \mathrm{mT}$ irrespective of the temperature. Comparing calculated $\left(1.6 \mu_{\mathrm{B}}\right)$ and measured $\left(0.29 \mu_{\mathrm{B}}\right)$ magnetic moments, it is evident that there are competing magnetic interactions in the system as a fully ferromagnetic state is not reached.

The results of this paper are important for research fields including, but not being limited to, MAX phases, since the theoretical predictions are experimentally verified for materials of novel compositions (a new MAX phase element - Mn) with new properties (magnetic MAX phases). The validated research approach can therefore most likely be used for materials of other structures and compositions. Furthermore, a new interesting ternary magnetic nanolaminate has been added to the magnetic materials family. 



\section{FUTURE RESEARCH}

Exploration of Ga-containing MAX phase thin film synthesis lead to the discovery of a new magnetic MAX phase $-\mathrm{Mn}_{2} \mathrm{GaC}$.

As was noted in the first publication about $\mathrm{Mn}_{2} \mathrm{GaC}$, the magnetic behaviour of the material is not fully understood and should be investigated further. There are many possible magnetic configurations for the ground state of this phase, as proposed from the theory. However synchrotron radiation as well as neutron diffraction analysis can shed light on the magnetic structure and indicated complex behaviour.

As $\mathrm{Mn}_{2} \mathrm{GaC}$ is a stable MAX phase, other alloying elements on the $M$ site could be studied, not being limited to the neighbouring Cr. As $\mathrm{Cr}$ itself exhibits significant local magnetic moment in the MAX phase structure, alloying $\mathrm{Mn}_{2} \mathrm{GaC}$ with a non-magnetic metal could give rise to different magnetic behaviour and other types of magnetic interactions. 



\section{BIBLIOGRAPHY}

[1] J.B. Carlson, Science 189753 (1975).

[2] P. Eklund, M. Beckers, U. Jansson, H. Högberg, L. Hultman, Thin Solid Films 518 $1851(2010)$.

[3] M.W. Barsoum, Prog. solid state chem. 28201 (2000).

[4] J. Rosen, M. Dahlqvist, S.I. Simak, D.R. McKenzie, M.M.M. Bilek, Appl. Phys. Lett. 97073103 (2010).

[5] W. Jeitschko, H. Nowotny, F. Benesovsky, Monatsh. Chem. 94844 (1963).

[6] M.W. Barsoum, T. El-Raghy, J. Am. Ceram. Soc. 791953 (1996).

[7] J. Emmerlich, H. Hogberg, S. Sasvari, P.O.A. Persson, L. Hultman, J.-P. Palmquist, U. Jansson, J.M. Molina-Aldareguia, Z. Czigany, J. Appl. Phys. 964817 (2004).

[8] J.P. Palmquist, S. Li, P.O.Å. Persson, J. Emmerlich, O. Wilhelmsson, H. Högberg, M.I. Katsnelson, B. Johansson, R. Ahuja, O. Eriksson, L. Hultman, U. Jansson, Phys. Rev. B 70165401 (2004).

[9] H. Högberg, P. Eklund, J. Emmerlich, J. Birch, L. Hultman, J. Mater. Res. 20779 (2005).

[10] S. Kuchida, T. Muranaka, K. Kawashima, K. Inoue, M. Yoshikawa, J. Akimitsu, Physica C: Superconductivity 49477 (2013).

[11] M. Naguib, M. Kurtoglu, V. Presser, J. Lu, J. Niu, M. Heon, L. Hultman, Y. Gogotsi, M.W. Barsoum, Adv. Mater. 234248 (2011).

[12] M.W. Barsoum, M. Radovic, Annu. Rev. Mater. Sci. 41195 (2011).

[13] J. Emmerlich, P. Eklund, D. Rittrich, H. Högberg, L. Hultman, J. Mater. Res. 222279 (2007).

[14] J. Emmerlich, G. Gassner, P. Eklund, H. Högberg, L. Hultman, Wear 264914 (2008).

[15] A. Mendoza-Galván, M. Rybka, K. Järrendahl, H. Arwin, M. Magnuson, L. Hultman, M.W. Barsoum, J. Appl. Phys. 109013530 (2011).

[16] A.S. Ingason, A. Mockute, M. Dahlqvist, F. Magnus, S. Olafsson, U.B. Arnalds, B. Alling, I.A. Abrikosov, B. Hjörvarsson, P.O.Å. Persson, J. Rosen, Phys. Rev. Lett. 110195502 (2013).

[17] A. Mockute, M. Dahlqvist, J. Emmerlich, L. Hultman, J.M. Schneider, P.O.Å. Persson, J. Rosen, Phys. Rev. B 87094113 (2013). 
[18] A. Mockute, P.O.Å. Persson, F. Magnus, A.S. Ingason, S. Olafsson, L. Hultman, J. Rosen, Phys. Status Solidi Rapid Res. Lett. 8420 (2014).

[19] L. Zheng, J. Wang, X. Lu, F. Li, J. Wang, Y. Zhou, J. Am. Ceram. Soc. 933068 (2010).

[20] Z. Lin, M. Zhuo, Y. Zhou, M. Li, J. Wang, J. Am. Ceram. Soc. 893765 (2006).

[21] J. Zhang, B. Liu, J.Y. Wang, Y.C. Zhou, J. Mater. Res. 2439 (2009).

[22] M.W. Barsoum, T. El - Raghy, L.U.J.T. Ogbuji, J. Electrochem. Soc. 1442508 (1997).

[23] M. Naguib, G.W. Bentzel, J. Shah, J. Halim, E.N. Caspi, J. Lu, L. Hultman, M.W. Barsoum, Mater. Res. Lett. 1 (2014).

[24] T. Cabioch, P. Eklund, V. Mauchamp, M. Jaouen, M.W. Barsoum, J. Eur. Ceram. Soc. 33897 (2013).

[25] M.W. Barsoum, T. El-Raghy, M. Ali, Metall. Mater. Trans. A 311857 (2000).

[26] J. Rosen, P.O.Å. Persson, M. Ionescu, A. Kondyurin, D.R. McKenzie, M.M.M. Bilek, Appl. Phys. Lett. 92064102 (2008).

[27] A. Mockute, M. Dahlqvist, L. Hultman, P.O.Å. Persson, J. Rosen, J Mater Sci 483686 (2013).

[28] M. Dahlqvist, B. Alling, I.A. Abrikosov, J. Rosén, Phys. Rev. B 81024111 (2010).

[29] M. Baben, L. Shang, J. Emmerlich, J.M. Schneider, Acta Mater. 604810 (2012).

[30] M. Dahlqvist, B. Alling, J. Rosén, Phys. Rev. B 81220102 (2010).

[31] P. Eklund, M. Dahlqvist, O. Tengstrand, L. Hultman, J. Lu, N. Nedfors, U. Jansson, J. Rosén, Phys. Rev. Lett. 109035502 (2012).

[32] M. Dahlqvist, B. Alling, I.A. Abrikosov, J. Rosen, Phys. Rev. B 84220403 (2011).

[33] A. Mockute, J. Lu, E.J. Moon, M. Yan, B. Anasori, S.J. May, M.W. Barsoum, J. Rosen, Mater. Res. Lett. 1 (2014).

[34] Q.Z. Tao, C.F. Hu, S. Lin, H.B. Zhang, F.Z. Li, D. Qu, M.L. Wu, Y.P. Sun, Y. Sakka, M.W. Barsoum, Mater. Res. Lett. 1 (2014).

[35] S. Lin, P. Tong, B.S. Wang, Y.N. Huang, W.J. Lu, D.F. Shao, B.C. Zhao, W.H. Song, Y.P. Sun, J. Appl. Phys. 113053502 (2013).

[36] W. Luo, R. Ahuja, J. Phys.-Condens. Mat. 20064217 (2008).

[37] M. Bode, M. Heide, K. von Bergmann, P. Ferriani, S. Heinze, G. Bihlmayer, A. Kubetzka, O. Pietzsch, S. Blugel, R. Wiesendanger, Nature 447190 (2007).

[38] R.J. McIntyre, Solar Energy Materials 785 (1982).

[39] P. Eklund, M. Beckers, J. Frodelius, H. Högberg, L. Hultman, J. Vac. Sci.Technol. A 251381 (2007). 
[40] J.E. Sundgren, B.O. Johansson, S.E. Karlsson, Thin Solid Films 105353 (1983).

[41] A. Khatibi, J. Palisaitis, C. Höglund, A. Eriksson, P.O.Å. Persson, J. Jensen, J. Birch, P. Eklund, L. Hultman, Thin Solid Films 5192426 (2011).

[42] G.B. Thompson, D.D. Allred, Journal of X-Ray Science and Technology 7159 (1997).

[43] T. Larsson, H.O. Blom, S. Berg, M. Östling, Thin Solid Films 172133 (1989).

[44] M. Beckers, N. Schell, R.M.S. Martins, A. Mücklich, W. Möller, L. Hultman, J. Appl. Phys. 102074916 (2007).

[45] S. Foner, Rev. Sci. Instrum. 30548 (1959).

[46] M. Dahlqvist, B. Alling, J. Rosen, J. Appl. Phys. 113216103 (2013). 



\section{Included Papers}

The articles associated with this thesis have been removed for copyright reasons. For more details about these see:

http://urn.kb.se/resolve?urn=urn:nbn:se:liu:diva- 110764 